\title{
Sensing Principle Analysis of FBG Based Sensors
}

\author{
Imran Khan ${ }^{1}$, Istiaq Ahmed ${ }^{2}$ \\ ${ }^{1}$ Department of EEE, Jessore Science \& Technology University, Jessore, Bangladesh \\ ${ }^{2}$ Department of ECE, Sylhet International University, Sylhet, Bangladesh
}

\begin{abstract}
In this paper the temperature and strain sensing principle of FBG based sensors are analyzed through experimental procedures. The property of the FBG changes due to the thermo-optic and elasto-optic effect which results the change of period of the gratings \& the effective refractive index respectively. Due to this FBG's property change results Bragg wavelength shift. From the measured Bragg wavelength shift with respect to the reference (at room temperature \& no strain applied) Bragg wavelength we can calculate the corresponding temperature or strain. For this type of temperature sensor the sensitivity found is (9.9 \pm 0.13$) \mathrm{pm} / \mathrm{C}$.
\end{abstract}

Keywords: Bragg wavelength, Effective refractive index, Elasto-optic effect, Fiber Bragg gratings, Thermooptic effect

\section{INTRODUCTION}

Specially designed Optical Fiber (OF) can be worked as a sensor. The OF for sensor application is designed in such a way so that there is a short portion in the fiber where the core refractive index is different from the usual fiber core and cladding refractive index [1]. Normally, a periodic structure is introduced in that short portion of the OF core. This portion of the fiber core reflects the light of a specific wavelength \& generally known as Fiber Bragg Gratings (FBG). A type of Distributed Bragg Reflector (DBR) constructed in a short segment of Optical Fiber that reflects the light of a particular wave length (known as Bragg wavelength) and transmit all others is known as FBG. Where DBR is a structure formed from multiple layers of alternating materials with varying refractive index, or by periodic variation of some characteristic (such as height) of a dielectric waveguide, resulting in periodic variation in the effective refractive index in the guide [2],[3]. A sensor whose sensitivity is based on the Bragg wavelength shift of the Fiber Bragg Gratings is known as FBG based sensors. In other way we can say that FBG is periodic wavelength scale variation of refractive index inscribed in the segment of the fiber core. Bragg gratings reflect the light at a particular wavelength which satisfies Bragg condition. This reflection in a grating occurs as coupling between forward and back propagation modes at certain wavelength take place [4]. The coupling coefficient of the modes is maximal when special condition (Bragg condition) between wave vectors of light and vector number of the grating is satisfied:

$$
m \cdot \lambda_{B}=2 \cdot n_{e f f} \cdot \Lambda
$$

$\lambda_{B}$ is wavelength of light called Bragg wavelength, $\Lambda$ is grating period, $n_{e f f}$ is effective refractive index of the core and $m$ is the diffraction order. The operation principle of fiber Bragg grating is illustrated in Fig. 1.

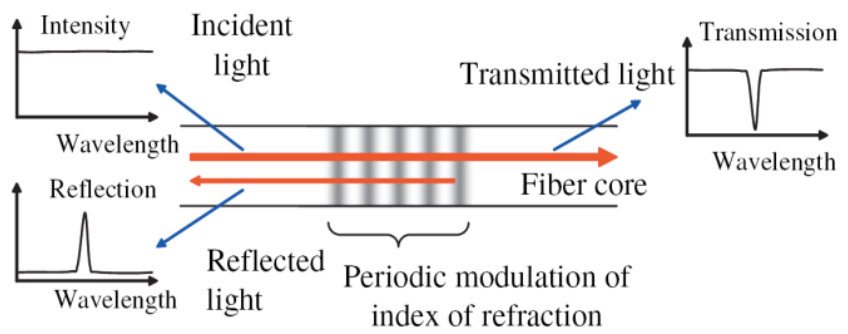

Fig. 1 Fiber Bragg Grating, refractive index modulation and spectral response [2]

For a single FBG theoretically there exists infinite number of Bragg wavelength. It can be clearly seen from the (1), as for different values of $m$, i.e. diffraction order Bragg wavelength are different. These Bragg wavelengths are separated from each other by quite large spectral range, so on practice only one (first or sometimes second) Bragg resonance wavelength is being used. For instance, when the first Bragg wavelength of the grating $(\mathrm{m}=1)$ is $1550 \mathrm{~nm}$, then the second one is twice less: $750 \mathrm{~nm}$. While the spectral range of sources used for fiber usually doesn't exceed $100 \mathrm{~nm}$. 
Additional Bragg peaks can occur if the modulation of the refractive index in FBG is not sinusoidal (which is usually the case). For instance in case of rectangular grating, the Fourier spectrum the latter has a number of modulation frequencies, which can results in several Bragg peaks. Even though most of the gratings inscribed in fiber has nearly sinusoidal index modulation. There are different structures of FBGs, in this paper the experiment and analysis was done on uniform FBG to analyze the sensing capability of a FBG as a sensor.

\section{SENSING PRINCIPLE}

In (1) two parameters can depend on external condition change, i.e. change of temperature and strain. These parameters are effective index of the core $\left(n_{\text {eff }}\right)$ and the period of the grating $(\Lambda)$. When temperature changes the effective index is changing due to thermo-optic effect, while the period changes due to thermal expansion of the glass. When strain is applied effective index is changing due to elasto-optic effect, while period is changing because of elasticity of the glass and can be explained by Hooke's law. As a result of strain and temperature change the effective index is changing by $\Delta n_{\text {eff }}$ and the period of the grating by $\Delta \Lambda$, which will result in overall Bragg wavelength change $\Delta \lambda_{B}$. So the Bragg condition will take the following form:

$\lambda_{B}+\Delta \lambda_{B}=2 \cdot\left(n_{\text {eff }}+\Delta n_{\text {eff }}\right) \cdot(\Lambda+\Delta \Lambda)$

$=2\left(n_{\text {eff }} \cdot \Lambda+n_{\text {eff }} \cdot \Delta \Lambda+\Lambda \cdot \Delta n_{\text {eff }}+\Delta n_{\text {eff }} \cdot \Delta \Lambda\right)$

The last term of the expression can be neglected as it is multiplication of two small quantities. Also taking into account (1) (unperturbed Bragg condition), we will come to the formula for the shift of Bragg wavelength:

$$
\Delta \lambda_{B}=2\left(n_{\text {eff }} \cdot \Delta \Lambda+\Lambda \cdot \Delta n_{\text {eff }}\right)
$$

Due to the change of any parameter mentioned above, the Bragg wavelength will shift. By observing the corresponding Bragg wavelength shift with the reference one can sense the change.

\section{TEMPERATURE SENSITIVITY: EXPERIMENTAL RESULTS AND ANALYSIS}

For this experiment - current used to heat up the grating was 0.8A (maximum). This current flow through steel metal plate and produce heat, the FBG was glued on this metal plate. One end of this FBG was connected to the signal generator and the other end was connected with an Optical Spectrum Analyser (OSA). Maximum temperature used was $51 \mathrm{C} \&$ maximum voltage used was $4.5 \mathrm{~V}$. Step used to increase current to increase temperature was $0.04 \mathrm{~A}$. Experimental results due to the temperature change are listed in the Table I [5].

Table I

Experimental Results of Temperature Change on FBG

\begin{tabular}{|c|c|c|c|}
\hline $\begin{array}{c}\text { No. of } \\
\text { Readings }\end{array}$ & $\begin{array}{c}\text { Current } \\
(\mathbf{A})\end{array}$ & $\begin{array}{c}\text { Temperature } \\
(\mathbf{C})\end{array}$ & $\begin{array}{c}\text { Wavelength } \\
(\mathbf{n m})\end{array}$ \\
\hline 1 & 0.00 & 24.1 & 1583.294 \\
\hline 2 & 0.04 & 24.4 & 1583.295 \\
\hline 3 & 0.08 & 25.1 & 1583.300 \\
\hline 4 & 0.12 & 25.7 & 1583.304 \\
\hline 5 & 0.16 & 26.4 & 1583.310 \\
\hline 6 & 0.20 & 27.2 & 1583.317 \\
\hline 7 & 0.24 & 28 & 1583.324 \\
\hline 8 & 0.28 & 28.8 & 1583.332 \\
\hline 9 & 0.32 & 29.7 & 1583.339 \\
\hline 10 & 0.36 & 30.8 & 1583.350 \\
\hline 11 & 0.40 & 32 & 1583.359 \\
\hline 12 & 0.44 & 33.1 & 1583.370 \\
\hline 13 & 0.48 & 34.5 & 1583.383 \\
\hline 14 & 0.52 & 35.6 & 1583.392 \\
\hline 15 & 0.56 & 37.2 & 1583.408 \\
\hline 16 & 0.60 & 38.7 & 1583.422 \\
\hline 17 & 0.64 & 43.4 & 1583.471 \\
\hline 18 & 0.78 & 45 & 1583.487 \\
\hline 19 & 0.72 & 47 & 1583.506 \\
\hline 20 & 0.76 & 48.8 & 1583.522 \\
\hline 21 & 0.80 & 51 & 1583.544 \\
\hline
\end{tabular}


From the above Table I we plot the data as Temperature vs. Bragg wavelength and we got almost linear curve (Fig. $2 \&$ Fig. 3). This implies that with the temperature change the Bragg wavelength shifts linearly. So by observing the Bragg wavelength shift with the reference Bragg wavelength we can sense the temperature through this sensor.

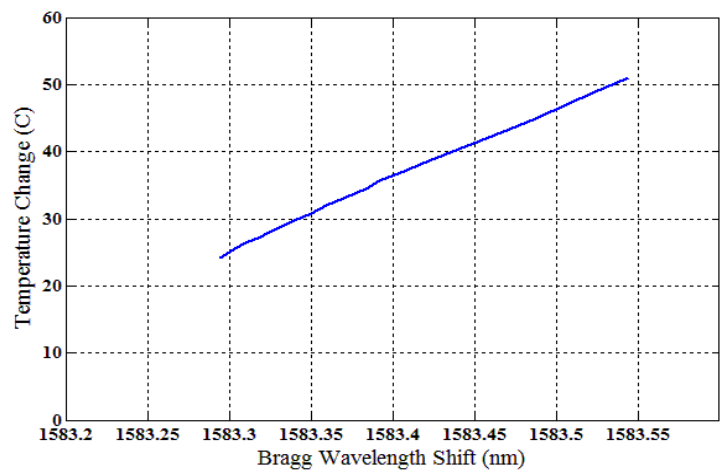

Fig. 2 Experimental results of the Bragg wavelength dependence on temperature [5].

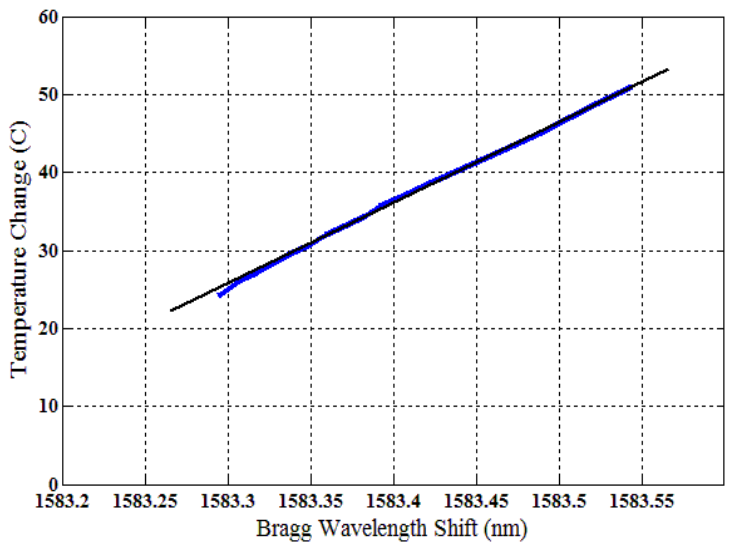

Fig. 3 Experimental results of the Bragg wavelength dependence on temperature and linear fitting.

\section{Calculation of Error}

For the calculation of the error [6] let us consider the measurements with readings 14 and 21.

Table II

Data for Error Calculation

\begin{tabular}{|c|c|c|}
\hline $\begin{array}{c}\text { No. of } \\
\text { Readings }\end{array}$ & $\begin{array}{c}\text { Temperature } \\
\text { (C) }\end{array}$ & $\begin{array}{c}\text { Wavelength } \\
\text { (nm) }\end{array}$ \\
\hline 14 & 35.6 & 1583.392 \\
\hline 21 & 51 & 1583.544 \\
\hline
\end{tabular}

The absolute error of the temperature is $0.1{ }^{0} \mathrm{C}$ and for wavelength 0.001 , so the sensitivity is:

$$
\begin{aligned}
& S=\frac{\left(\lambda_{21} \pm \Delta \lambda_{\text {err }}\right)-\left(\lambda_{14} \pm \Delta \lambda_{\text {err }}\right)}{\left(T_{21} \pm \Delta T_{\text {err }}\right)-\left(T_{14} \pm \Delta T_{\text {err }}\right)}=\frac{\left(\lambda_{21}-\lambda_{14}\right) \pm \Delta \lambda_{\text {dif_err }}}{\left(T_{21}-T_{14}\right) \pm \Delta T_{\text {dif_err }}} \\
& =\frac{0.152}{15.4}+\Delta S_{\text {err }}=0.0099+\Delta S_{\text {err }}
\end{aligned}
$$

Where the errors from the difference can be calculated from absolute errors by: 


$$
\begin{aligned}
& \Delta \lambda_{\text {dif }_{-} \text {err }}=\sqrt{\left(\Delta \lambda_{\text {err }}\right)^{2}+\left(\Delta \lambda_{\text {err }}\right)^{2}}=\sqrt{2\left(\Delta \lambda_{\text {err }}\right)^{2}} \\
& =\sqrt{2(0.001)^{2}}=0.0014 \\
& \Delta T_{\text {dif }_{-} \text {err }}=\sqrt{\left(\Delta T_{\text {err }}\right)^{2}+\left(\Delta T_{\text {err }}\right)^{2}}=\sqrt{2\left(\Delta T_{\text {err }}\right)^{2}} \\
& =\sqrt{2(0.1)^{2}}=0.14
\end{aligned}
$$

Error generated from the ratio can be calculated in the following way:

$$
\begin{aligned}
& \Delta S_{\text {err }}=S \cdot \sqrt{\left(\frac{\Delta \lambda_{\text {dif_err }}}{\Delta \lambda}\right)^{2}+\left(\frac{\Delta T_{\text {dif_err }}}{\Delta T}\right)^{2}} \\
& =0.0099 \cdot \sqrt{\left(\frac{0.0014}{0.152}\right)^{2}+\left(\frac{0.14}{15.4}\right)^{2}}=0.00013 \mathrm{~nm} / \mathrm{C}
\end{aligned}
$$

$$
\Delta S_{\text {err }}=0.13 \mathrm{pm} / \mathrm{C}
$$

So the sensitivity of the sensor with the calculated error is:

$(9.9 \pm 0.13) \mathrm{pm} / \mathrm{C}$

\section{AXIAL STRAIN SENSITIVITY: EXPERIMENTAL RESULTS AND ANALYSIS}

For optical fiber maximum strain that can be tolerated is $0.1 \%$ of its length. The length of our OF $=40$ $\mathrm{cm}$. So the maximum strain that can be applied in terms of distance is $0.4 \mathrm{~mm}$.

We plotted data from Table III and got again almost linear curve for this strain change on the FBG (Fig. 4 \& Fig. 5).

Table III

Experimental Results of Strain Change on FBG

\begin{tabular}{|c|c|c|}
\hline $\begin{array}{c}\text { No. of } \\
\text { Readings }\end{array}$ & $\begin{array}{c}\text { Distance } \\
(\mathbf{m m})\end{array}$ & $\begin{array}{c}\text { Wavelength } \\
\text { (nm) }\end{array}$ \\
\hline 1 & 0.00 & 1583.300 \\
\hline 2 & 0.02 & 1583.310 \\
\hline 3 & 0.04 & 1583.356 \\
\hline 4 & 0.06 & 1583.415 \\
\hline 5 & 0.08 & 1583.474 \\
\hline 6 & 0.1 & 1583.536 \\
\hline 7 & 0.12 & 1583.594 \\
\hline 8 & 0.14 & 1583.654 \\
\hline 9 & 0.16 & 1583.715 \\
\hline 10 & 0.18 & 1583.769 \\
\hline 11 & 0.20 & 1583.839 \\
\hline 12 & 0.22 & 1583.894 \\
\hline 13 & 0.24 & 1583.956 \\
\hline 14 & 0.26 & 1584.020 \\
\hline 15 & 0.28 & 1584.077 \\
\hline 16 & 0.30 & 1584.140 \\
\hline 17 & 0.32 & 1584.199 \\
\hline 18 & 0.34 & 1584.269 \\
\hline 19 & 0.36 & 1584.317 \\
\hline 20 & 0.38 & 1584.336 \\
\hline 21 & 0.40 & 1584.428 \\
\hline & & \\
\hline
\end{tabular}




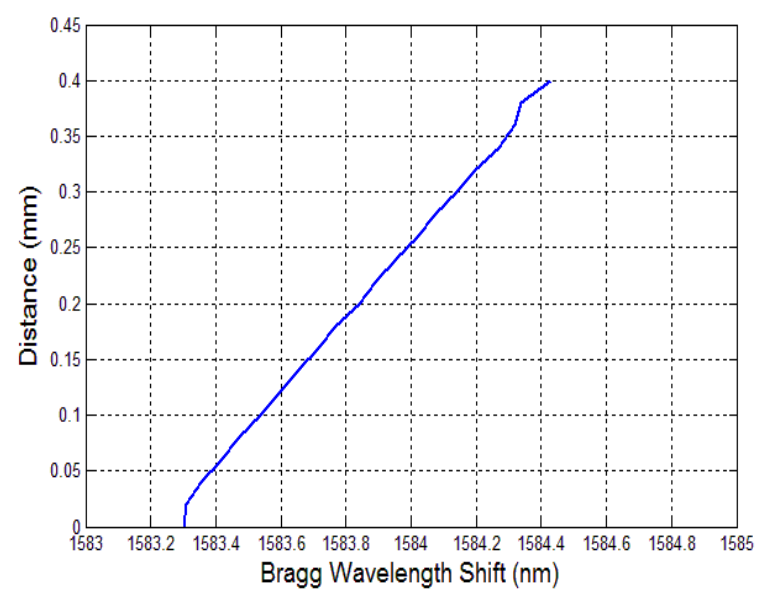

Fig. 4 Experimental results of the Bragg wavelength dependence on strain.

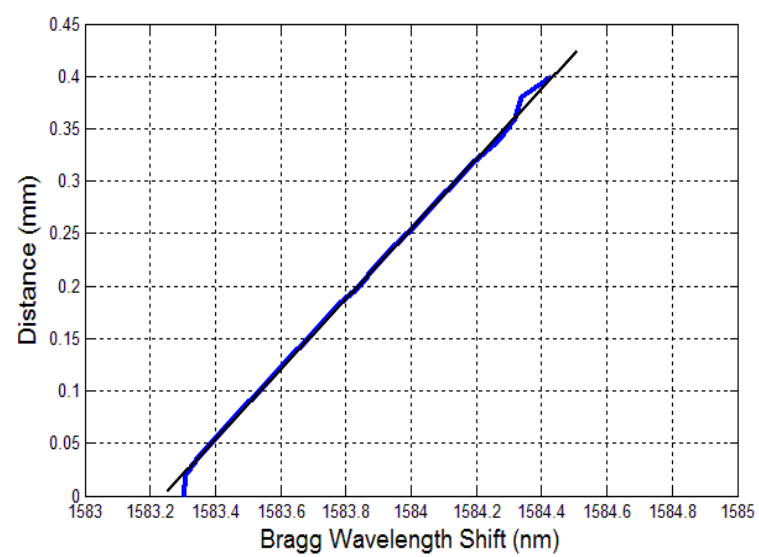

Fig. 5 Experimental results of the Bragg wavelength dependence on strain and linear fitting.

From the experimental results we can see that due to the strain change the Bragg wavelength shifts linearly with respect to the applied strain. The above curve is not completely linear at the beginning and at the end because of the experimental setup and the range respectively. When the experimental setup becomes stable with the surroundings the curve becomes linear in response of the strain. So by observing the Bragg wavelength shift (Fig.6) with respect to the reference Bragg wavelength we can sense the strain on the FBG.

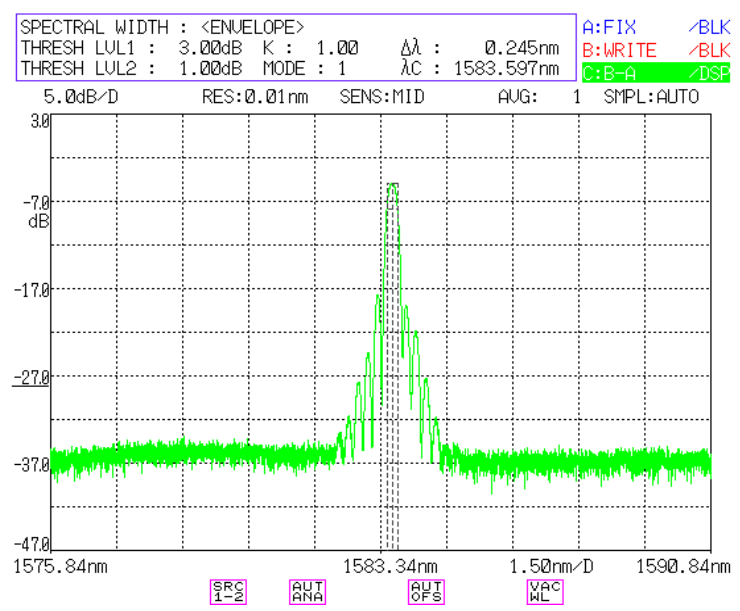

Fig. 6 Observed reflected Bragg wavelength in OSA [5]. 


\section{APPLICATION OF FBG BASED SENSORS}

Based on the application certain FBGs are chosen to make the sensors. FBG based sensors have a lot of applications. This type of strain sensors can be used in civil engineering work such as river bridge safety monitoring, telecommunication and other tower stability monitoring. Now a day a lot of bridges over river or canal are built in many developing countries (such as Bangladesh) but there is no sensor included with the bridge. As a result it is not possible to check the bridge stability or safety. As example, a crack was found on the Jamuna Bridge in Bangladesh which was not an old bridge [7]. This type of crack was occurred due to the overloaded vehicle let pass over the bridge. And the bridge has no sensor to monitor this extra mechanical stress and strain. This kind of damage of the bridge can be easily prevented. From the above experimental results and analysis we know that FBG based sensors are sensitive to the mechanical strain as well as stress. If the strain is too high due to the overloaded vehicle on the bridge then the sensor will automatically response or ring the alarm. The FBG based temperature sensors can be used in industry or any other places where accurate temperature reading with negligible error is required.

\section{CONCLUSION}

It is observed and analysed that when there is a change in optical properties of a material (FBG) because of heat radiation the effective refractive index (as well as the grating period) is changing as a result the Bragg wavelength shifts. Again a change in the refractive index of an optical fiber caused by variation in the length or width of the fiber core in response to mechanical stress results the change of effective refractive index and causes the Bragg wavelength shift according to (1). There is a linear relationship between the Bragg wavelength shift and the temperature [8] as well as the strain [9] change. So by comparing the shifted Bragg wavelength due to the temperature or stress with the reference Bragg wavelength at room temperature and without any stresses, it is possible to sense the temperature and strain. So it is verified by lab experiment that FBG based sensors are good sensor to sense very small quantity accurately with negligible error. Thus FBG can work as a sensor. Even chirped Fiber Bragg Grating can also be used as sensors [10].

\section{ACKNOWLEDGEMENTS}

The authors acknowledge the support by Photonics Department, Vrije Universiteit Brussel (VUB). Work at the laboratory was supported by TONA. The authors would like to thank Tigran Baghdasaryan, Yassin Chowdhury and Prof. Francis Berghmans for their support.

\section{REFERENCES}

[1] E.Udd, Fiber Optic Sensors: An Introduction for Engineerings and Scientists (John Wiley and Sons, New York, 1991).

[2] A.Cusano, A. Cutolo and M. Giordano, "Fiber Bragg Gratings Evanescent Wave Sensors: A View Back and Recent Advancements", Sensors, Springer-Verlag Berlin Heidelberg, 2008.

[3] K.O.Hill and G. Meltz, "Fiber Bragg Grating Technology Fundamentals and Overview", Journal of Lightwave Technology, Vol. 15, No. 8, August 1997

[4] G.P. Agrawal, Fiber-Optic Communication Systems, (John Wiley \& Sons, 2002).

[5] Imran Khan, "Optical Fiber based Microwaves Sensor Using Surface Plasmon Resonance", Proceedings of International Conference on Informatics, Electronics \& Vision, ISSN: 2226-2105, pp. 310-315, May, 2012.

[6] http://home.clara.net/rod.beavon/err_comb.htm.

[7] The Daily Star, Cracks develop as overloaded trucks let pass over it, 17-01 -2008.

[8] H.Meng,W.Shen,G.Zhang, C.Tan and X. Huang, "Fiber Brag grating-based fiber sensor for simultaneous measurement of refractive index and temperature", Sensors and Actuators B: Chemical, 150 (2010), pp. 226-229.

[9] A.Mendez, "Fiber Bragg grating sensors: a market overview", Proceedings of SPIE, vol. 6619, 661905 (2007).

[10] Y.Okabe, R. Tsuji and N. Takeda, "Application of chirped Fiber bragg grating sensors for identification of crack locations in composites", Composites: Part A 35, pp-59-65, 2004. 